\title{
A randomised controlled trial comparing adductor canal block and femoral nerve block for knee arthroplasty
}

\author{
Yean Chin $\underline{\mathrm{Lim}}^{1}$, mBBs, MMed, How Yow Kelvin $\underline{\text { Quek }}{ }^{1}$, MBBS, MMed, Wai Heng Jimmy $\underline{\text { Phoo }}^{1}$, MBBS, MMed,
} Chou Liang Mah $^{1}$, MBBS, MMed, Shumei $\underline{\operatorname{Tan}}^{2}$, BPhysio(Hons)

INTRODUCTION Adductor canal block (ACB) is hypothesised to provide superior analgesia to femoral nerve block (FNB) for total knee arthroplasty (TKA) while preserving quadriceps strength.

METHODS 30 patients undergoing TKA were randomised to receive either ACB or FNB. Baseline tests of quadriceps strength were performed. Ultrasound-guided blocks with $30 \mathrm{~mL}$ of $0.5 \%$ ropivacaine were administered before induction of general anaesthesia. Patient-controlled analgesia (morphine) was prescribed for postoperative analgesia. The primary outcome of this prospective, double-blinded, randomised controlled trial was morphine consumption (mean \pm standard deviation) in the first 24 hours. Secondary outcomes were pain scores using a numeric rating scale (median and interquartile range [IQR]), quadriceps strength (\% of baseline) and functional outcomes at 24 hours and 48 hours postoperatively.

RESULTS There was no statistically significant difference in morphine consumption at 24 hours between the ACB and FNB groups ( $21 \pm 11 \mathrm{mg}$ vs. $20 \pm 12 \mathrm{mg} ; \mathrm{p}=0.85$ ). No statistically significant differences were observed between the ACB and FNB groups in pain scores at 24 hours (at rest: 0 [IQR 0-2] vs. 0 [IQR 0-2]; on movement: 5 [IQR 4-8] vs. 5 [IQR $3-8]$ ) and quadriceps strength ( 24 hours: $28.8 \% \pm 26.1 \%$ vs. $26.8 \% \pm 19.6 \%$ of baseline; 48 hours: $31.5 \pm 23.1 \%$ vs. $33.7 \%$ $\pm 20.1 \%$ of baseline). There were also no statistically significant differences in functional outcomes and length of stay. CONCLUSION We found no statistically significant differences in analgesic effects, quadriceps strength or functional recovery postoperatively between ACB and FNB.

Keywords: analgesia, nerve block, total knee arthroplasty

\section{INTRODUCTION}

Total knee arthroplasty (TKA) is associated with significant postoperative pain. Multimodal analgesia techniques are employed to provide patient comfort and facilitate early mobilisation. Although the femoral nerve block (FNB) provides good analgesia for TKA, ${ }^{(1-3)}$ it results in quadriceps weakness. ${ }^{(4)}$ There are also concerns that peripheral nerve blocks may be related to falls. ${ }^{(5,6)}$

Compared to FNB, the adductor canal block (ACB) is performed more distally in the adductor canal, which is bounded by the sartorius muscle above, the vastus medialis muscle laterally and the adductor muscles medially. ACB, which is postulated to block the saphenous nerve, nerve to vastus medialis and posterior branches of the obturator nerve, ${ }^{(7)}$ has been shown to preserve quadriceps strength and ability to ambulate better than FNB in healthy volunteers. ${ }^{(8,9)}$ It is hypothesised that patients who receive $A C B$ will have less motor weakness than those receiving FNB following TKA, as the motor nerves supplying the quadriceps muscles, besides the vastus medialis, would have branched off. A study comparing continuous ACB and FNB in patients who underwent TKA under spinal anaesthesia showed that patients who received ACB had better quadriceps strength, with no difference in morphine consumption and pain scores. ${ }^{(10)}$ In another study comparing patients who underwent TKA under combined spinal-epidural, patients in the ACB group had better quadriceps strength at 6-8 hours, but no differences in quadriceps strength, pain scores or morphine consumption were demonstrated at 24 hours and 48 hours. ${ }^{(11)}$

To date, no study has compared single-shot ACB and FNB in patients undergoing TKA under general anaesthesia instead of central neuraxial blockade. We aimed to study the analgesic effect and quadriceps strength in patients receiving single-shot ACB versus FNB for TKA. The primary outcome is morphine consumption in the first 24 hours. Secondary outcomes are pain scores at 24 and 48 hours, morphine consumption at 48 hours, postoperative nausea/vomiting, sedation scores, quadriceps strength and functional outcomes.

\section{METHODS}

This prospective, randomised, double-blinded, controlled study was approved by the SingHealth Centralised Institutional Review Board (2013/921/D) in December 2013. The trial was registered with ClinicalTrials.gov (NCT02033603). This study was conducted at Changi General Hospital in accordance to guidelines for Good Clinical Practice and the Declaration of Helsinki. After obtaining written, informed consent, 30 patients undergoing primary TKA were recruited from February 2014 to September 2014.

Inclusion criteria were patients aged $45-85$ years old who were scheduled for primary TKA under general anaesthesia due to primary osteoarthritis, with American Society of Anesthesiologists (ASA) physical status $1-3$ and a body mass index (BMI) of

${ }^{1}$ Department of Anaesthesia and Surgical Intensive Care, ${ }^{2}$ Department of Rehabilitative Services, Changi General Hospital, Singapore

Correspondence: Dr Yean Chin Lim, Consultant, Department of Anaesthesia and Surgical Intensive Care, Changi General Hospital, 2 Simei Street 3 , Singapore 529889. lim.yean.chin@singhealth.com.sg 
18-35 kg/m². Exclusion criteria were patients who: (a) were unable to give consent; (b) had regular consumption of strong opioids (morphine, oxycodone) or steroids; (c) had allergy to local anaesthetics or any drugs included in the study; (d) had lower limb surgery in the preceding year; or (e) had pre-existing neurological deficits. Computer-generated block randomisation with 1:1 ratio and blocks of ten was used. Randomisation was concealed in opaque envelopes. The patient, primary anaesthetist and data collector were blinded. Unfortunately due to the nature of the study, the proceduralist could not be blinded.

On the day of admission and prior to surgery, baseline quadriceps strength and functional outcomes were assessed. Quadriceps strength was assessed as maximum voluntary isometric contraction using a handheld dynamometer, which is considered a reliable instrument for measuring muscle strength. ${ }^{(12)}$ The patient was seated with knees flexed at $90^{\circ}$ and the handheld dynamometer was fixed against the anterior surface of the tibia with straps. They were instructed to take two seconds to reach maximum effort, and then maintain it for three seconds before relaxing. The average of three attempts was recorded.

Functional outcome was measured using the Timed Up and Go test (TUG) and the 30-second Chair Stand Test (30s-CST). TUG is a reliable, validated tool for rehabilitation of orthopaedic patients ${ }^{(13)}$ and records the time taken for the patient to get up from a chair, walk $3 \mathrm{~m}$ and return to the chair. 30s-CST assesses the number of times a patient is able to stand up from a chair in 30 seconds and has been shown to reflect lower body strength in community-dwelling older adults. ${ }^{(14)}$

Premedication of oral paracetamol $1 \mathrm{~g}$ was given half an hour prior to surgery. The block was performed by one of the study investigators. Intravenous access was obtained and sedation with midazolam (up to $5 \mathrm{mg}$ ) was allowed. Standard monitors were applied and supplementary oxygen was provided during the block. Either an FNB or ACB was performed under real-time ultrasonography guidance using a SonoSite M-Turbo ultrasound machine (FujiFilm, Japan) with linear probe (HFL38x, 13-6 MHz). A short-axis, in-plane technique was used. A Stimuplex ${ }^{\circledR}$ A100 (B Braun, Melsungen, Germany) 21-gauge needle was used and $30 \mathrm{~mL}$ of $0.5 \%$ ropivacaine was administered. For FNB, the injection was performed at the level of the inguinal ligament, before the bifurcation of the artery. The needle approach was from lateral to medial, and local anaesthetic was deposited circumferentially around the nerve. For ACB, the block was performed at mid-thigh, midway between the anterior superior iliac spine and patella. The needle approach was from lateral to medial, and local anaesthetic was deposited laterally, above and medially to the artery, between the vaso-adductor membrane and femoral artery. The site of injection and volume administered were based on those of previous studies. ${ }^{(8,10,15)}$ Block success was tested half an hour after the block was performed by assessing patient's sensation to cold over the medial aspect of the lower leg.

General anaesthesia was induced with intravenous fentanyl (up to $2 \mathrm{mcg} / \mathrm{kg}$ ) and propofol (1-3 mg/kg). A supraglottic device was inserted to maintain the airway and anaesthesia was maintained on an oxygen/air/sevoflurane mixture. Intravenous morphine (up to $0.2 \mathrm{mg} / \mathrm{kg}$ ) was administered intraoperatively for analgesia as required. The total amount of intraoperative opioids (fentanyl/ morphine) used was recorded. Intravenous ondansetron $4 \mathrm{mg}$ was given at the end of the operation for antiemesis. Postoperative analgesia included patient-controlled analgesia morphine with no basal infusion, bolus $1 \mathrm{mg}$, five-minute lock-out time and maximum of $12 \mathrm{mg}$ /hour. Oral paracetamol $1 \mathrm{~g}$ was administered six-hourly and etoricoxib $120 \mathrm{mg}$ was given once daily for three days. In the recovery area, intravenous morphine (up to $0.2 \mathrm{mg} / \mathrm{kg}$ ) was administered to achieve pain control before discharge to the ward.

The primary endpoint was morphine consumption in the first 24 hours (including morphine administered in recovery and via patient-controlled analgesia). Secondary outcomes included pain scores at rest and on movement at 24 and 48 hours, morphine consumption at 48 hours, postoperative nausea/vomiting, sedation scores, quadriceps strength and functional outcomes.

Pain scores, assessed using a numeric rating scale (0-10), were recorded at one hour postoperatively in recovery, at rest. At 24 and 48 hours postoperatively, pain scores were recorded in the ward, at rest and during knee flexion. Nausea was defined as an unpleasant sensation associated with the urge to vomit, and vomiting was defined as the forceful expulsion of gastric contents from the mouth. The presence/absence of nausea and the number of episodes of vomiting (more than $10 \mathrm{~mL}$ ) were recorded. The use of antiemetic was also recorded.

Sedation was assessed on a four-point scale $(0=$ no sedation, 1 = light, 2 = moderate, 3 = severe). Quadriceps strength assessment (percentage of baseline), TUG and 30s-CST were performed at 24 and 48 hours postoperatively. We assessed for early complications related to peripheral nerve block, such as haematoma, infection, neurological deficits and falls, at 48 hours.

A study performed in the local population showed mean morphine consumption of $27 \mathrm{mg}$ in patients who received an $\mathrm{FNB}$, using $30 \mathrm{~mL}$ of $0.5 \%$ ropivacaine in the first 24 hours post TKA, with a standard deviation (SD) of $9 \mathrm{mg} .{ }^{(16)}$ We considered a difference of morphine consumption of $10 \mathrm{mg}$ to be clinically significant. With a power of $80 \%$ and two-tailed p-value of 0.05 , we would require 13 patients in each group. We aimed to recruit 30 patients to account for possible drop-outs.

Data was analysed using IBM SPSS Statistics version 21.0 (IBM Corp, Armonk, NY, USA). Categorical outcomes were analysed with chi-square or Fisher's exact test when the sample was small. Ordinal data and other non-parametric data, such as pain scores, TUG and 30s-CST, were analysed with Mann-Whitney $U$ test and presented as median and interquartile range. Continuous data, such as morphine consumption and quadriceps strength, was analysed with the $t$-test and presented as mean \pm SD. All comparisons between FNB and ACB were two-sided, and a p-value $<0.05$ was considered statistically significant.

\section{RESULTS}

Patients were enrolled from February 2014 to September 2014. Patient recruitment and flow are described in the CONSORT (Consolidated Standards of Reporting Trials) diagram (Fig. 1). All patients received the intervention that they were randomised to 


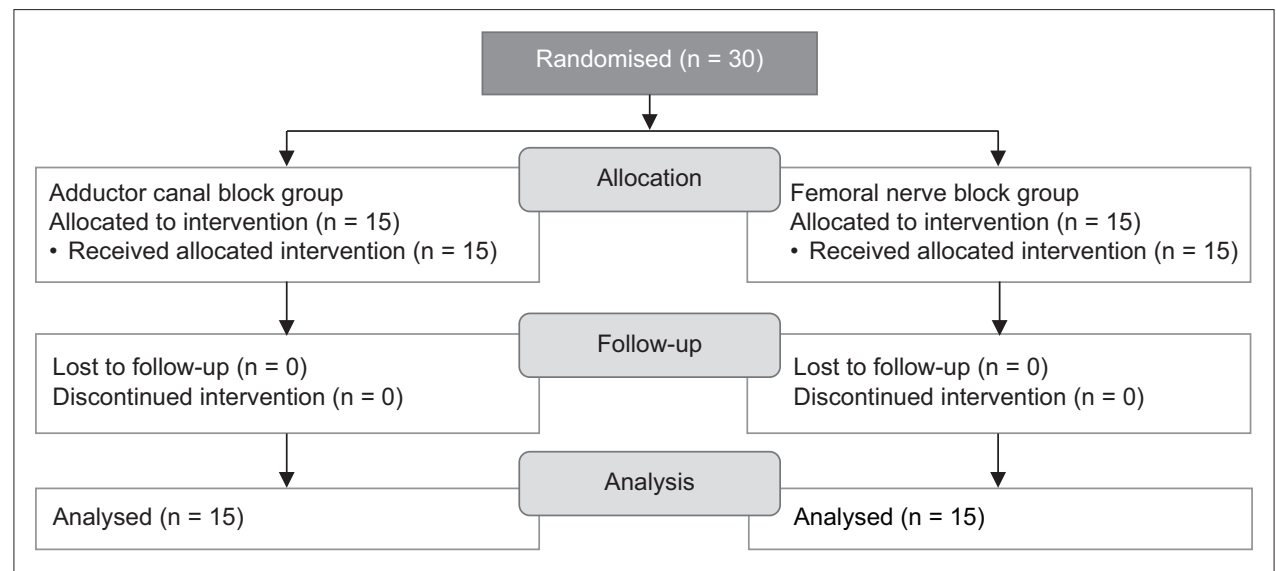

Fig. 1 CONSORT diagram shows patient recruitment and flow.

Table I. Characteristics and perioperative data of patients who received adductor canal block (ACB) or femoral nerve block (FNB) for total knee arthroplasty.

\begin{tabular}{|c|c|c|}
\hline \multirow[t]{2}{*}{ Parameter } & \multicolumn{2}{|c|}{ No. (\%) } \\
\hline & $A C B(n=15)$ & FNB $(n=15)$ \\
\hline Age* $^{*}(y r)$ & $63 \pm 7$ & $65 \pm 8$ \\
\hline \multicolumn{3}{|l|}{ Gender } \\
\hline Male & $5(33)$ & $7(47)$ \\
\hline Female & $10(67)$ & $8(54)$ \\
\hline$B M I *\left(k g / m^{2}\right)$ & $26.6 \pm 4.3$ & $28.0 \pm 3.5$ \\
\hline \multicolumn{3}{|l|}{ ASA status } \\
\hline 1 & $1(7)$ & 0 \\
\hline 2 & $12(80)$ & $11(73)$ \\
\hline 3 & $2(13)$ & $4(27)$ \\
\hline \multicolumn{3}{|l|}{ Regular preoperative analgesics } \\
\hline Paracetamol & $1(3)$ & $2(7)$ \\
\hline NSAIDs & $2(7)$ & 0 \\
\hline \multicolumn{3}{|l|}{ Pain scores $^{\dagger}$} \\
\hline At rest & $0(0-2)$ & $0(0-5)$ \\
\hline On movement & $3(3-5)$ & $3(2-5)$ \\
\hline Baseline quadriceps strength* (N) & $107.6 \pm 41.4$ & $115.1 \pm 41.4$ \\
\hline
\end{tabular}

* Data presented as mean \pm standard deviation. †Data presented as median (interquartile range). ASA: American Society of Anesthesiologists; BMI: body mass index; NSAIDs: nonsteroidal anti-inflammatory drugs

receive, and there was no loss to follow-up or withdrawal. All blocks were successful and all the 30 patients recruited were included in the analysis.

Patient characteristics and preoperative data are presented in Table I. There were no statistically significant differences in demographics between the two groups in terms of age, gender, $\mathrm{BMI}$ and ASA status. There were no differences in regular consumption of simple analgesics preoperatively and no patients had regular consumption of weak opioids. Preoperative pain scores and quadriceps strength were similar in both groups.

Perioperative use of opioids is shown in Table II. There were no statistically significant differences in intraoperative opioids administered. No fentanyl was administered to patients in recovery, and the amount of morphine administered in recovery
Table II. Perioperative usage of opioids in the adductor canal block (ACB) and femoral nerve block (FNB) groups.

\begin{tabular}{|clll|}
\hline Opioid & \multicolumn{2}{c}{ Mean \pm SD } & p-value \\
\cline { 2 - 3 } & ACB $(\mathbf{n}=\mathbf{1 5})$ & FNB $(\mathbf{n}=\mathbf{1 5})$ & \\
\hline Fentanyl $(\mathbf{m c g})$ & & $110 \pm 28$ & 0.13 \\
\hline Intraoperative & $98 \pm 6$ & 0 & - \\
\hline In recovery & 0 & & \\
\hline Morphine $(\mathbf{m g})$ & & $8 \pm 2$ & 0.29 \\
\hline Intraoperative & $7 \pm 2$ & $0 \pm 1$ & 0.50 \\
\hline In recovery & $0 \pm 2$ & & \\
\hline
\end{tabular}

SD: standard deviation

was similar in both groups. There was no statistically significant difference in tourniquet time (ACB group $105.7 \pm 15.2$ minutes vs. FNB group $108.3 \pm 27.2$ minutes; $p=0.743$ ). There were no statistically significant differences in morphine consumption at 24 and 48 hours (24 hours: ACB group $21 \pm 11 \mathrm{mg}$ vs. FNB group $20 \pm 12 \mathrm{mg}, \mathrm{p}=0.85 ; 48$ hours $\mathrm{ACB}$ group $41 \pm 22 \mathrm{mg}$ vs. FNB group $39 \pm 29 \mathrm{mg}, \mathrm{p}=0.83$ ).

Secondary outcomes of pain scores at rest and on movement are shown in Table III; no statistically significant differences in pain scores at one hour, 24 hours and 48 hours postoperatively were observed between the groups. Morphine-related side effects are presented in Table IV; there were no statistically significant differences in nausea, vomiting and level of sedation. Quadriceps strength at 24 and 48 hours are displayed in Fig. 2; no statistically significant difference in quadriceps strength was observed. Quadriceps strength was $28.8 \% \pm 26.1 \%$ versus $26.8 \% \pm 19.6 \%$ of baseline $(p=0.84)$ at 24 hours, and $31.5 \% \pm 23.1 \%$ versus $33.7 \% \pm 20.1 \%$ of baseline $(p=0.79)$ at 48 hours in the ACB and FNB groups, respectively.

There were no statistically significant differences in the functional outcomes of TUG and 30s-CST. At 24 hours, TUG was 69.5 (IQR 44.7-122.3) seconds in the ACB group and 72 (IQR 66.5-116.4) seconds in the FNB group $(p=0.69)$. At 48 hours, TUG was 67.8 (IQR 45.9-74.3) seconds in the ACB group and 71.2 (IQR 45.5-86.2) seconds in the FNB group $(p=0.57)$. 30s-CST was 8 (IQR 7-9) versus $6($ IQR 3-7) at 24 hours $(\mathrm{p}=0.06)$ and $7(\mathrm{IQR} 4-9)$ versus $8(\mathrm{IQR} 4-9)$ at 48 hours 
Table III. Postoperative pain scores of patients in adductor canal block (ACB) and femoral nerve block (FNB) groups.

\begin{tabular}{|llll|}
\hline \multirow{2}{*}{ Pain score } & \multicolumn{2}{c}{ Median (IQR) } & \multirow{2}{*}{ p-value } \\
\cline { 2 - 3 } & ACB (n= 15) & FNB (n= 15) & \\
\hline 1 hour, at rest & $2(0-5)$ & $2(0-3)$ & 1.00 \\
\hline 24 hours, at rest & $0(0-2)$ & $0(0-2)$ & 0.54 \\
\hline 24 hours, on movement & $5(4-8)$ & $5(3-8)$ & 0.78 \\
\hline 48 hours, at rest & $0(0-0)$ & $0(0-0)$ & 1.00 \\
\hline 48 hours, on movement & $3(2-5)$ & $3(2-5)$ & 0.37 \\
\hline
\end{tabular}

IQR: interquartile range

Table IV. Morphine-related side effects of patients the adductor canal block (ACB) and femoral nerve block (FNB) groups.

\begin{tabular}{|llll|}
\hline \multirow{2}{*}{$\begin{array}{l}\text { Side-effect at } \\
\mathbf{2 4} \text { hours }\end{array}$} & \multicolumn{2}{c}{ Median (IQR)/no. (\%) } & p-value \\
\cline { 2 - 3 } & ACB $(\mathbf{n}=\mathbf{1 5})$ & FNB (n=15) & \\
\hline Nausea & $0(0-0)$ & $0(0-0)$ & 0.78 \\
\hline Vomiting & $0(0-1)$ & $0(0-2)$ & 0.44 \\
\hline Sedation & & & 1.00 \\
\hline None & $15(100)$ & $14(93)$ & \\
\hline Mild & $0(0)$ & $1(7)$ & \\
\hline
\end{tabular}

IQR: interquartile range

$(p=0.73)$ in the ACB and FNB groups, respectively. The time to achieve $30 \mathrm{~m}$ walk is similar in the two groups, i.e. 2 (IQR 2-3) days; $p=0.84)$. The length of stay was 4 (IQR 4-5) days in the ACB group versus 4 (IQR 3-6) days in the FNB group $(p=0.57$ ). There were no early complications (e.g. bleeding, infection, neurapraxia and falls) related to peripheral nerve blockades.

\section{DISCUSSION}

To our knowledge, this is the first randomised controlled trial comparing ACB with FNB in patients who underwent TKA under general anaesthesia instead of central neuraxial blocks. ${ }^{(10,11)}$ Performing blocks preoperatively allows us to test the success of the block as well as compare intraoperative opioid consumption. The use of general anaesthesia without any muscle relaxant, as compared to central neuraxial blocks, makes the measurement of quadriceps strength and functional outcomes more reliable.

Our study showed that there were no statistically significant differences in the analgesic effects produced by the two blocks. The perioperative morphine consumption and pain scores at one, 24 and 48 hours postoperatively were similar between the groups. Likewise, Kim et al's study, which compared ACB with FNB in patients undergoing TKA under combined spinalepidural, showed that $A C B$ was not inferior to FNB in terms of pain scores and opioid consumption. ${ }^{(11)}$ Comparing continuous $\mathrm{ACB}$ with continuous $\mathrm{FNB}$ in patients undergoing TKA under spinal anaesthesia, Jaeger et al's study also found no statistical differences in morphine consumption and pain scores. ${ }^{(10)}$ These results are consistent with our findings and suggest that, although ACB is not superior to FNB for analgesia following TKA, its analgesic effect may be comparable to that of FNB.

One of the side effects of FNB is quadriceps weakness. ${ }^{(4)}$ $\mathrm{ACB}$ has been proposed to have a quadriceps-sparing effect, as

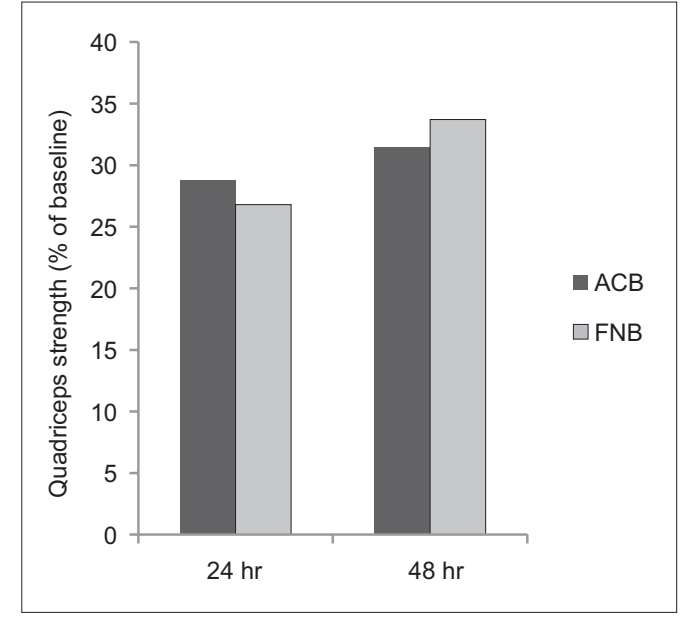

Fig. 2 Chart shows quadriceps strength of patients receiving adductor canal block (ACB) or femoral nerve block (FNB) at 24 and 48 hours postoperatively.

the block is performed distal to where the motor fibres of the femoral nerve (except for the nerve to the vastus medialis) have branched off. ${ }^{(7)}$ Both Jaeger et $\mathrm{al}^{(8)}$ and Kwofie et al, ${ }^{(9)}$ whose studies were performed in healthy volunteers, showed preservation of quadriceps strength in those who received $A C B$ as opposed to those who received FNB. These promising results have led to studies comparing these two blocks in patients undergoing TKA. Jaeger et al's study reported quadriceps strength of $52 \%$ of baseline in patients with continuous ACB and $18 \%$ in patients with continuous FNB. ${ }^{(10)}$ In Kim et al's study, higher quadriceps strength at 6-8 hours was demonstrated in patients receiving single-shot ACB compared to those who received single-shot FNB, although no difference was demonstrable at 24 and 48 hours. ${ }^{(11)}$

Similarly, we were unable to demonstrate any statistically significant differences in quadriceps strength at 24 and 48 hours postoperatively. Quadriceps strength at 6-8 hours was not measured in our study; this was one of the limitations of our study. In a pilot study, we had found that patients in our institution were unable to comply with the assessment of quadriceps strength at six hours postoperatively for various reasons (including sedation, giddiness and pain). As our institutional practice is for patient ambulation to take place on postoperative Day 1 , any difference in quadriceps strength at 6-8 hours postoperatively may, therefore, not translate to any clinical difference.

We opine that the results in healthy volunteers cannot be extrapolated to patients undergoing surgery, as there are other factors resulting in quadriceps weakness postoperatively besides regional anaesthesia technique. For instance, the use of a tourniquet can lead to axonal compression, electromyography changes, as well as delays in nerve conduction ${ }^{(17,18)}$ and time to straight leg-raising. ${ }^{(19)}$ All surgeons in our institution routinely use a tourniquet when performing TKA. Quadriceps strength has been demonstrated to decrease by $60 \%-62 \%$ in patients post TKA, possibly due to failure of voluntary muscle activation. ${ }^{(20,21)}$

Jaeger et al's study ${ }^{(10)}$ showed a difference in quadriceps strength at 24 hours between those who received ACB and those who received FNB; however, this did not translate to any 
difference in ability to mobilise, as measured by TUG. Similarly, our study found no statistically significant differences in functional outcomes, including TUG and 30s-CST. We also observed no statistically significant difference in the number of days required to achieve $30 \mathrm{~m}$ walk or time to discharge. There were no inpatient falls in our study. Despite concerns that FNB may increase the risk of falls, a recent review conducted in 191,570 patients showed that there was no association between peripheral nerve blocks and inpatient falls following TKA. ${ }^{(22)}$

There are some limitations to our study. The sample size was relatively small compared to that of other studies, although it was adequately powered for the primary outcome of the study. Also, the study was performed in a single centre and surgeries were performed by three different surgeons. Thus, variations in surgical techniques may have resulted in differing intensity of postoperative pain, opioid requirements and functional recovery.

There are also potential issues associated with the use of $\mathrm{ACB}$, including the risk of myositis, ${ }^{(23)}$ neuropathy and infection. Preoperative injection of local anaesthetic in the adductor canal, coupled with the compressive effects of the tourniquet and resultant ischaemia increases the risk of neuropathy. As the injection site for $\mathrm{ACB}$ is closer to the operative site compared to $\mathrm{FNB}$, this may pose a slightly increased risk of infection. Several recent studies on $\mathrm{ACB},{ }^{(24-26)}$ published after the completion of our study, have redefined the ACB and suggested that by performing the injection midway between the anterior superior iliac spine and patella as described by earlier studies, ${ }^{(8,10,15)}$ we are depositing the local anaesthetic into the femoral triangle instead of the adductor canal. As such, the technique that we have used in this study might be more accurately termed a 'femoral triangle block', which covers the saphenous nerve, nerve to vastus medialis and the medial femoral cutaneous nerve. If a large volume of injectates is used, it is possible that the injectate may spread proximally or distally. In contrast, injection in the adductor canal would reliably block the saphenous and, in a small proportion of patients, the medial retinacular nerve and posterior branch of the obturator nerve as well. The reason we were unable to demonstrate a difference in quadriceps strength could be due to the fact that the site of injection was proximal to the adductor canal and the injectates might have spread proximally as we had used a large volume of local anaesthetic.

In conclusion, the present study did not find any statistically significant differences in analgesic effects, quadriceps strength, functional outcomes or length of stay between the ACB and FNB groups. Current evidence is probably insufficient to support a change of practice from performing single-shot FNB to ACB at the level of the mid-thigh for TKA, unless future trials demonstrate sparing of quadriceps weakness in postoperative patients that translate to better functional recovery.

\section{ACKNOWLEDGEMENTS}

The study received funding from Changi General Hospital, Singapore. The research grant was used for the employment of a research assistant and purchase of the dynamometer.

\section{REFERENCES}

1. Allen HW, Liu SS, Ware PD, Nairn CS, Owens BD. Peripheral nerve blocks improve analgesia after total knee replacement surgery. Anesth Analg 1998; 87:93-7.

2. Wang $\mathrm{H}$, Boctor $\mathrm{B}$, Verner J. The effect of single-injection femoral nerve block on rehabilitation and length of hospital stay after total knee replacement. Reg Anesth Pain Med 2002; 27:139-44.

3. Paul JE, Arya A, Hurlburt L, et al. Femoral nerve block improves analgesia outcomes after total knee arthroplasty: a meta-analysis of randomized controlled trials. Anesthesiology 2010; 113:1144-62.

4. Charous MT, Madison SJ, Suresh PJ, et al. Continuous femoral nerve blocks: varying local anesthetic delivery method (bolus versus basal) to minimize quadriceps motor block while maintaining sensory block. Anesthesiology 2011; 115:774-81.

5. Ilfeld BM, Duke KB, Donohue MC. The association between lower extremity continuous peripheral nerve blocks and patient falls after knee and hip arthroplasty. Anesth Analg 2010; 111:1552-4.

6. Muraskin SI, Conrad B, Zheng N, Morey TE, Enneking FK. Falls associated with lower-extremity-nerve blocks: a pilot investigation of mechanisms. Reg Anesth Pain Med 2007; 32:67-72.

7. Lund J, Jenstrup MT, Jaeger P, Sørensen AM, Dahl JB. Continuous adductorcanal-blockade for adjuvant post-operative analgesia after major knee surgery: preliminary results. Acta Anaesthesiol Scand 2011; 55:14-9.

8. Jaeger $\mathrm{P}$, Nielsen ZJ, Henningsen $\mathrm{MH}$, et al. Adductor canal block versus femoral nerve block and quadriceps strength: a randomized, double-blind, placebo-controlled, crossover study in healthy volunteers. Anesthesiology 2013; 118:409-15.

9. Kwofie MK, Shastri UD, Gadsden JC, et al. The effects of ultrasound-guided adductor canal block versus femoral nerve block on quadriceps strength and fall risk: a blinded, randomized trial of volunteers. Reg Anesth Pain Med 2013; 38:321-5.

10. Jaeger P, Zaric D, Fomsgaard JS, et al. Adductor canal block versus femoral nerve block for analgesia after total knee arthroplasty: a randomized, doubleblind study. Reg Anesth Pain Med 2013; 38:526-32.

11. Kim DH, Lin Y, Goytizolo EA, et al. Adductor canal block versus femoral nerve block for total knee arthroplasty: a prospective, randomized, controlled trial. Anesthesiology 2014; 120:540-50.

12. Stark T, Walker B, Phillips JK, Fejer R, Beck R. Hand-held dynamometry correlation with the gold standard isokinetic dynamometry: a systematic review. PM R 2011; 3:472-9.

13. Yeung TS, Wessel J, Stratford PW, MacDermid JC. The timed up and go test for use on an inpatient orthopaedic rehabilitation ward. J Orthop Sports Phys Ther 2008; 38:410-7.

14. Jones CJ, Rikli RE, Beam WC. A 30-s chair-stand test as a measure of lower body strength in community-residing older adults. Res Q Exerc Sport 1999; 70:113-9.

15. Jenstrup MT, Jæger $\mathrm{P}$, Lund J, et al. Effects of adductor-canal-blockade on pain and ambulation after total knee arthroplasty: a randomized study. Acta Anaesthesiol Scand 2012; 56:357-64.

16. Ng HP, Cheong KF, Lim A, Lim J, Puhaindran ME. Intraoperative single-shot "3-in-1" femoral nerve block with ropivacaine $0.25 \%$, ropivacaine $0.5 \%$ or bupivacaine $0.25 \%$ provides comparable 48 -hr analgesia after unilateral total knee replacement. Can J Anaesth 2001; 48:1102-8.

17. Saunders KC, Louis DL, Weingarden SI, Waylonis GW. Effect of tourniquet time on postoperative quadriceps function. Clin Orthop Relat Res 1979; (143):194-9.

18. Estebe JP, Davies JM, Richebe P. The pneumatic tourniquet: mechanical, ischaemia-reperfusion and systemic effects. Eur J Anaesthesiol 2011; 28:404-11.

19. Abdel-Salam A, Eyres K. Effects of tourniquet during total knee arthroplasty. A prospective randomised study. J Bone Joint Surg Br 1995; 77:250-3.

20. Mizner RL, Petterson SC, Stevens JE, Vandenborne K, Snyder-Mackler L. Early quadriceps strength loss after total knee arthroplasty. The contributions of muscle atrophy and failure of voluntary muscle activation. J Bone Joint Surg Am 2005; 87:1047-53.

21. Stevens JE, Mizner RL, Snyder-Mackler L. Quadriceps strength and volitional activation before and after total knee arthroplasty for osteoarthritis. J Orthop Res 2003; 21:775-9.

22. Memtsoudis SG, Danninger T, Rasul R, et al. Inpatient falls after total knee arthroplasty: the role of anesthesia type and peripheral nerve blocks. Anesthesiology 2014; 120:551-63.

23. Neal JM, Salinas FV, Choi DS. Local anesthetic-induced myotoxicity after continuous adductor canal block. Reg Anesth Pain Med 2016; 41:723-7.

24. Bendtsen TF, Moriggl B, Chan V, Pedersen EM, Børglum J. Defining adductor canal block. Reg Anesth Pain Med 2014; 39:253-4.

25. Bendtsen TF, Moriggl B, Chan V, Pedersen EM, Børglum J. Redefining the adductor canal block. Reg Anesth Pain Med 2014; 39:442-3.

26. Bendtsen TF, Moriggl B, Chan V, Børglum J. The optimal analgesic block for total knee arthroplasty. Reg Anesth Pain Med 2016; 41:711-9. 